\title{
SPECT-CT image fusion could enhance Meckel scan
}

\section{Letter to the Editor}

$\mathrm{M}$ eckel's diverticulum is the most common congenital abnormality of the gastrointestinal tract. Complications include obstruction, intussusception, perforation, diverticulitis, and gastrointestinal hemorrhage. Despite availability of modern imaging techniques, its diagnosis does not follow a proposed sequence and remains delayed. ${ }^{[1] 99 \mathrm{~m}} \mathrm{Tc}$ pertechnetate scintigraphy can help to detect ectopic gastric mucosa in the abdomen, ${ }^{[2]}$ depending on the location and size of the ectopic tissue. The number of false negative findings could be reduced using image fusion as an appropriate technique.

We report a 14-month-old girl presenting with black stools and hematochezia who had a lowered hemoglobin level of $5.6 \mathrm{mg} / \mathrm{dl}$. Abdominal ultrasound and gastrointestinal endoscopy revealed no pathology. Contrast enhanced magnetic resonance tomography finding remained non-specific and inconclusive. Nuclear medicine was consulted to rule out or confirm a Meckel's diverticulum. Detection of ectopic gastric mucosa was attempted using intravenous injection of 79 $\mathrm{MBq}{ }^{99 \mathrm{~m}} \mathrm{Tc}$ pertechnetate after blocking the thyroid with triiodothyronine. Sequential planar scintigraphy during 30 minutes revealed a weak and small abdominal focus after 25 minutes (one image per minute). This focus projected on the urinary bladder (Fig. 1). Single Photon Emission Computer Tomography (SPECT) imaging was acquired and co-registered with a simultaneously

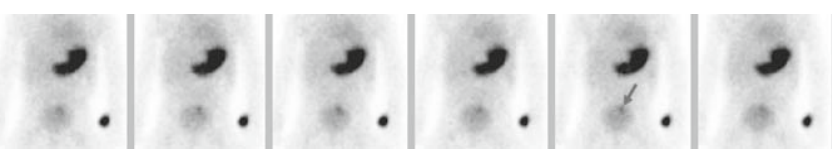

Fig. 1. Sequential planar scintigraphy shows a focus projected on the urinary bladder (sequence 25-30 minutes post injection).
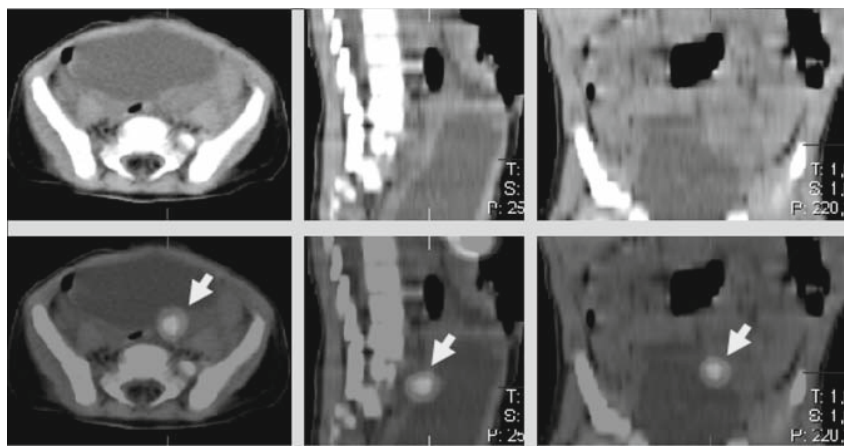

Fig. 2. SPECT/CT in 3 dimensions locates a lesion suspected as Meckel's diverticulum. acquired low-dose CT scan on a hybrid machine (Fig. 2), showing the lesion craniodorsal of the urinary bladder in an area of small intestines. Subsequently, the patient was referred to surgery within 3 hours. Laparatomy revealed a Meckel's diverticulum in a small intestine loop, which was consecutively resected and histologically investigated. Ectopic gastric mucosa showing chronic inflammation was confirmed as the source of the bleeding. SPECT/CT was the key method allowing targeted surgery in the patient who completely recovered thereafter.

The ${ }^{99 \mathrm{~m}}$ Tc pertechnetate scan causes a radiation exposure far below $0.05 \mathrm{mSv}$ effective dose, being a small fraction of a regular thyroid scan dose. Attenuation correction CT has an exposure of additionally $2 \mathrm{mSv}$, which is below the annual exposure from natural sources. This severely contrasts with the suggestion to use high resolution $\mathrm{CT}$ in the first line, ${ }^{[3]}$ because its radiation exposure of 10 to $20 \mathrm{mSv}$ is considerably higher. We suggest that simultaneous acquisition of SPECT and low dose attenuation CT could add a valuable means to localize a suspected Meckel's diverticulum prior to gastrointestinal endoscopy and laparoscopy. The diagnostic sensitivity reported to be $65 \%{ }^{[3]}$ mainly results from the relatively low signal to noise relation in planar scintigraphy. Provided SPECT/CT image fusion is readily available, it may significantly enhance diagnostic outcome as our example clearly shows.

Peter Schneider, Christian Düren, Christoph Reiners, Clinic and Polyclinic for Nuclear Medicine University Clinic of Würzburg, Oberdürrbacherstrasse 6, 97080 Würzburg, Germany Email: Schneider_p@klinik.uni-wuerzburg.de

\section{References}

1 Kiratli PO, Aksoy T, Bozkurt MF, Orhan D. Detection of ectopic gastric mucosa using $99 \mathrm{mTc}$ pertechnetate: review of the literature. Ann Nucl Med 2009;23:97-105.

2 Dolezal J, Vizda J. Experiences with detection of the ectopic gastric mucosa by means of Tc-99m pertechnetate disodium scintigraphy in children with lower gastrointestinal bleeding. Eur J Pediatr Surg 2008;18:258-260.

3 Olson DE, Kim YW, Donnelly LF. CT findings in children with Meckel's diverticulum. Pediatr Radiol 2009;39:659-663.

doi:10.1007/s12519-010-0227-7 